\section{Survival after rectal cancer: differences between hospital catchment areas. A nationwide study in Sweden}

\author{
P Blomqvist, A Ekbom, O Nyrén, U B Krusemo, R Bergström, H-O Adami
}

it is still uncertain whether such treatment should be centralised to large units or not. Advocates of centralisation have argued that greater experience on the part of the surgical team will improve the preoperative and perioperative staging, maximise local tumour control, reduce postoperative mortality, and allow more frequent use of sphincter-saving procedures. Reviews on the relation between hospital volume and outcome have recently been published. ${ }^{12}$ Briefly, the evidence for benefits of centralisation is not compelling, but comparisons between hospitals may be confounded by differences in case mix. Many studies are hampered by being focused mainly on in hospital mortality, by using non-uniform definitions of procedures, and by the fact that the volumes of operations performed are sometimes expressed either by hospital or by physician. Moreover, most studies are hospital based and many use only short term follow up.

Recent trials have shown the benefit of preoperative radiotherapy. ${ }^{3-5}$ Clinical trials for comparison of different approaches to the organisation of surgical services and/or the effects of different levels of experience among surgeons would be unfeasible and probably unethical. Observational studies may therefore offer the only realistic alternative for analysing differences in survival in rectal cancer between categories of hospitals and temporal trends.

Sweden offers an ideal setting for studying these issues for several reasons. Hospitalprovided medical services are population based and referable to defined catchment areas within each county. All patients with a diagnosed cancer can be readily identified from the nationwide Cancer Registry, and the individually unique national registration numbers allow complete long term follow up of survival by record linkages.

We took advantage of these prerequisites to study differences in survival between rectal cancer patients from catchment areas of hospitals that represent different volumes of rectal cancer surgery and different degrees of specialisation. We postulated that after standardisation for age and sex, there is little variation between catchment area populations with regard to the average stage or severity of the disease at which symptoms arise and prompt the patient to contact health care. Hence, as the availability of medical services is uniform over Sweden, any differences in survival between catchment areas can be attributed to differences in management or the combined quality of diagnostic and therapeutic efforts. 


\section{Subjects and methods}

SETTING

Sweden has a public health care system based on county councils. When in-hospital care is required, the patient normally first attends the hospital responsible for the catchment area in which he or she lives. Only after referral from that hospital, or in cases of emergency, are the patients admitted to hospitals outside their catchment area.

There are four different categories of hospital. Small local hospitals $(\mathrm{n}=48)$ have catchment areas comprising 30000 to 100000 inhabitants, and large local hospitals $(n=19)$ have 100000 or more. County hospitals $(\mathrm{n}=$ 23) serve 100 000-250 000 people, and in addition they receive referrals from local hospitals in the same county. Regional/ university hospitals ( $n=10$ ) have larger catchment areas, and are also referral centres for county hospitals. Thus these four categories reflect increasing volumes of admissions and operations, and successively more specialised care. There was no organised centralisation of colorectal cancer surgery during the course of our study.

\section{STUDY COHORT}

Our study cohort consisted of all living persons with a rectal cancer diagnosis (ICD-7, 154) notified to the $98 \%$ complete nationwide Swedish Cancer Registry from 1 January 1973 to 31 December $1992 .{ }^{6}$ We did not include cases discovered incidentally at autopsy. About $97 \%$ of the cases were verified histologically. The register contained information on date of birth, date of diagnosis, domicile (county, municipality, parish), and hospital that reported the malignancy, but not on tumour stage or treatment given. On the basis of the domicile noted in the register and detailed information about the catchment area boundaries obtained from the county councils, each cancer case was assigned to one hospital catchment area-that is, the catchment area in which he/she lived at the time of the rectal cancer diagnosis.

FOLLOW UP

Record linkage to the nationwide Register of Causes of Death, using the individually unique national registration numbers as identifiers, yielded information about dates of death up to the end of 1992. Thus the follow up ended on 31 December 1992, and patients not recorded in the Register of Causes of Death up to that date were considered to be alive.

STATISTICAL METHODS

The observed survival rates were calculated according to the actuarial (life table) method. ${ }^{7}$

Table 1 Characteristics of the newly diagnosed patients with rectal cancer in Sweden 1973-1992, by hospital catchment area category

\begin{tabular}{llllll}
\hline & $\begin{array}{l}\text { Regional/ } \\
\text { university }\end{array}$ & County & Large local & Small local & Total \\
\hline Number of cases & 6733 & 10212 & 6378 & 7488 & 30811 \\
Percentage of total & 21.9 & 33.1 & 20.7 & 24.3 & 100.0 \\
Mean age at diagnosis (y) & 69.5 & 70.0 & 69.9 & 69.9 & 69.9 \\
Median age at diagnosis (y) & 71 & 71 & 71 & 71 & 71 \\
Age $\geqslant 70$ at diagnosis (\%) & 54.8 & 56.8 & 55.8 & 55.3 & 55.8 \\
\hline
\end{tabular}

Relative survival was computed as the ratio of the observed survival among the cohort to the expected survival among individuals in the general population of the same sex, age (in five year age groups), and calendar year of observation. $^{89}$

We performed multivariate analyses on the entire cohort to estimate the independent effects of hospital catchment area category and co-variates such as age, calendar year of diagnosis, and duration of follow up. The analysis was based on grouped annual data and on a generalisation of the proportional hazards model, in which the rectal cancer specific hazard rate was assumed to be additive to the hazard rate in the general population. ${ }^{1011}$ This model was formulated as a generalised linear model, and estimates were obtained by the maximum likelihood method using the GLIM statistical software. ${ }^{12}$ We used categorised explanatory variables. In addition to models in which main effects were analysed, models with interaction terms were also estimated.

The estimated models were compared with respect to deviance, which describes the fit of the model. A deviance of the same order as the degrees of freedom is normally taken as an indication of a reasonable fit. However, in applications with a large number of cases in each cell, the variability of observed cases is often greater than that indicated by the Poisson or binomial theory. We used the difference in deviance in combination with the pattern and size of the variable estimates as an indicator of the quality of the different models. The results are given as relative hazards of dying from rectal cancer, with $95 \%$ confidence intervals.

\section{Results}

Our cohort consisted of 30811 rectal cancer patients, of whom $50.2 \%$ were men and $49.8 \%$ women. Table 1 shows their distribution between the catchment area categories and some baseline characteristics. The relative distribution over categories varied little during the study period 1973 to 1992 inclusive (data not shown). Over the two decades, the mean age at diagnosis rose from 69.4 to 71.3 years and the corresponding median age from 71 to 72 years. There were only minor intercategory differences in the age distribution. Catchment areas of regional hospitals had a mean of 34 newly diagnosed cases of rectal cancer per area and year, county hospitals 22, large local hospitals 17 , and small local hospitals eight.

We compared the category of the catchment area assigned to each patient on the basis of domicile with the corresponding category based on the hospital that reported the rectal cancer to the Cancer Registry, assuming that the latter was the hospital that in fact treated the patient. In $86.2 \%$ of the cases, these categories were identical. The cases notified from a hospital of a higher category than that of their home catchment area only constituted $6.1 \%$, typically referred from smaller to larger hospitals. These patients were, on average, younger than the average rectal cancer patient, whereas patients reported from a lower 
Table 2 One and five year relative survival after rectal cancer diagnosis by hospital catchment area category and time period of diagnosis

\begin{tabular}{|c|c|c|c|c|c|c|c|c|c|c|}
\hline & \multicolumn{5}{|c|}{ One year relative survival } & \multicolumn{5}{|c|}{ Five year relative survival } \\
\hline & Regional & County & Large local & Small local & All categories & Regional & County & Large local & Small local & All categories \\
\hline $1973-76$ & 0.70 & 0.68 & 0.70 & 0.68 & 0.69 & 0.39 & 0.38 & 0.41 & 0.37 & 0.39 \\
\hline $1977-80$ & 0.74 & 0.73 & 0.73 & 0.69 & 0.72 & 0.45 & 0.44 & 0.45 & 0.42 & 0.44 \\
\hline $1981-84$ & 0.74 & 0.76 & 0.74 & 0.73 & 0.74 & 0.46 & 0.50 & 0.46 & 0.45 & 0.47 \\
\hline $1985-88$ & 0.80 & 0.76 & 0.76 & 0.74 & 0.76 & 0.53 & 0.50 & 0.48 & 0.46 & 0.49 \\
\hline 1989-92 & 0.80 & 0.80 & 0.78 & 0.76 & 0.79 & - & - & - & - & - \\
\hline $1973-92$ & 0.76 & 0.75 & 0.74 & 0.72 & 0.74 & 0.48 & 0.47 & 0.46 & 0.43 & 0.46 \\
\hline
\end{tabular}

category than that of their home catchment area $(4.3 \%)$ were slightly older. A small group of even older patients $(3.4 \%)$ were notified to the Cancer Registry from nursing homes.

Relative survival differed between catchment area categories (table 2). One year after diagnosis, the relative survival rates were $76 \%$ and $72 \%$ for rectal cancer patients living in catchment areas of regional hospitals and small local hospital respectively $(\mathrm{p}<0.001)$. The corresponding rates five years after diagnosis were $48 \%$ and $43 \% \quad(\mathrm{p}<0.001)$. The other two catchment area categories exhibited relative survival rates below the rates observed in catchment areas of regional hospitals, but clearly above those in areas served by small local hospitals. Differences in prognosis between catchment area categories were already noted one month after diagnosis. At that time, the difference in relative survival between catchment areas of regional and small local hospitals was one percentage point, and the order of rank between the catchment areas was equal to the orders observed one and five years after diagnosis (data not shown).

Figures 1 and 2 depict the one year and five year relative survival rates respectively by catchment area category and calendar year of diagnosis. Over the study period, the relative survival improved considerably in all catchment area categories, but the survival advantage among patients living in catchment areas of county and regional hospitals persisted.

Referrals may have had the result that hospitals of certain categories treated more severely ill cases than others. To shed light on the importance of this redistribution of patients, we also analysed relative survival according to the category of hospital that reported the case to the Cancer Registry. The differences in relative survival between categories of reporting hospitals were slightly smaller than between categories of catchment areas. The one year relative survival among patients

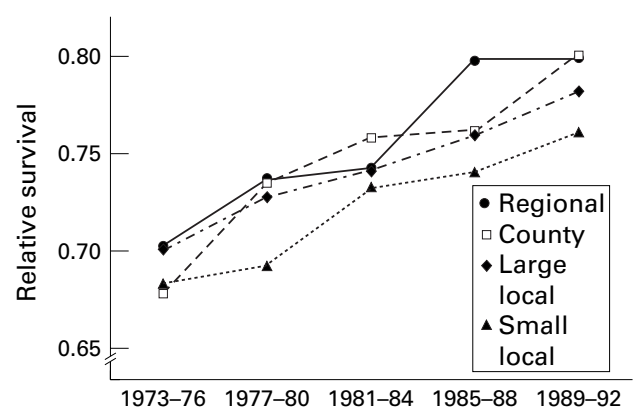

Figure 1 Rectal cancer in Sweden in the period 1973 to 1992. Relative one year survival by catchment area category. reported by regional/university hospitals was $76 \%$, compared with $75 \%$ among those reported by county or large local hospitals, and $73 \%$ among those reported by small local hospitals. The differences in five year relative survival between hospital categories were almost negligible. Patients reported to the Cancer Registry from a higher hospital category than was indicated by domicile-that is, patients likely to have been referred-had virtually the same one year relative survival as patients whose categories were identical, but their five year relative survival was the lowest of all subsets analysed.

According to the death certificates recorded in the Registry of Causes of Death, colorectal cancer accounted for $72 \%$ of all deaths in our cohort, while ischaemic heart disease and all other diseases of the circulatory system accounted for $9 \%$ and $6 \%$ respectively. There were only small differences in recorded causes of death between catchment area categories.

The survival advantage for patients living in catchment areas of regional/university hospitals, relative to those served by small local hospitals, remained statistically significant after adjustment for age at diagnosis, calendar period of diagnosis, and duration of follow up in multivariate analyses (table 3 ). The relative hazard of dying from the illness during the first five years after a rectal cancer diagnosis was $15 \%$ higher among patients living in catchment areas of small local hospitals. The considerable trendwise reduction in death risk with calendar period of diagnosis, and the increased risk among patients older than 70 years also persisted after these adjustments. The relative hazard of dying fell successively with each year that passed after the initial diagnosis. After having survived the first few years, the risk of dying during the later part of the follow up period was only a small fraction of the risk in the first year.

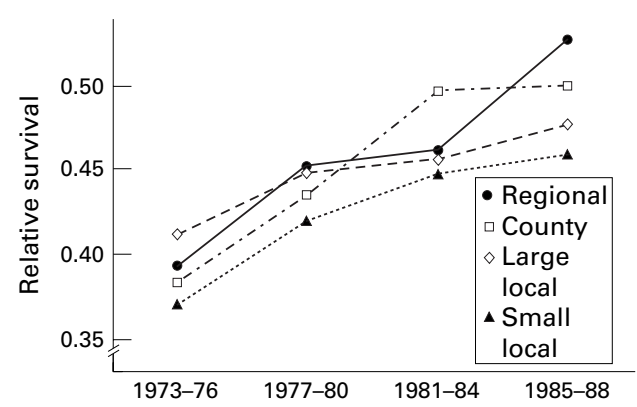

Figure 2 Rectal cancer in Sweden in the period 1973 to 1992. Relative five year survival by catchment area category. 
Table 3 Relative hazards (RH) of dying from rectal cancer, with 95\% confidence intervals (CI), based on the first five years of follow up

\begin{tabular}{|c|c|c|c|c|}
\hline \multirow[b]{2}{*}{ Variable } & \multicolumn{2}{|c|}{$\begin{array}{l}\text { Mulivariate analysis with } \\
\text { adjustment for follow up year }\end{array}$} & \multicolumn{2}{|c|}{$\begin{array}{l}\text { Multivariate main effects } \\
\text { model }\end{array}$} \\
\hline & $R H$ & $95 \% C I$ & $R H$ & $95 \% C I$ \\
\hline \multicolumn{5}{|l|}{ Catchment area category } \\
\hline Regional & 1.00 & Reference & 1.00 & Reference \\
\hline County & 1.04 & 0.93 to 1.15 & 1.03 & 0.97 to 1.10 \\
\hline Large local & 1.07 & 0.95 to 1.21 & 1.06 & 0.99 to 1.14 \\
\hline Small local & 1.15 & 1.03 to 1.29 & 1.15 & 1.07 to 1.22 \\
\hline \multicolumn{5}{|l|}{ Period of diagnosis } \\
\hline 1973-76 & 1.00 & Reference & 1.00 & Reference \\
\hline $1977-80$ & 0.88 & 0.80 to 0.98 & 0.88 & 0.82 to 0.94 \\
\hline $1981-84$ & 0.80 & 0.72 to 0.89 & 0.79 & 0.73 to 0.84 \\
\hline $1985-88$ & 0.74 & 0.67 to 0.82 & 0.73 & 0.68 to 0.78 \\
\hline $1989-92$ & 0.69 & 0.61 to 0.77 & 0.67 & 0.62 to 0.72 \\
\hline \multicolumn{5}{|l|}{ Age at diagnosis (y) } \\
\hline$\leqslant 69$ & 1.00 & Reference & 1.00 & Reference \\
\hline$\geqslant 70$ & 1.39 & 1.31 to 1.47 & 1.41 & 1.35 to 1.48 \\
\hline \multicolumn{5}{|l|}{ Follow up year } \\
\hline 1 & 1.00 & Reference & 1.00 & Reference \\
\hline 2 & 0.65 & 0.59 to 0.71 & 0.65 & 0.62 to 0.69 \\
\hline 3 & 0.48 & 0.43 to 0.55 & 0.49 & 0.46 to 0.53 \\
\hline 4 & 0.34 & 0.28 to 0.39 & 0.34 & 0.31 to 0.38 \\
\hline 5 & 0.27 & 0.22 to 0.33 & 0.28 & 0.25 to 0.31 \\
\hline
\end{tabular}

We found a strong interaction effect between age at diagnosis and duration of follow up (data not shown). This indicates that the excess risk of dying during the first follow up years was particularly high among older patients. Addition of a term for interaction between calendar period of diagnosis and catchment area category improved the model only marginally (reduction in deviance 21.8 on 12 degrees of freedom, $p=0.04)$. Hence, multivariate modelling provided no strong support for the impression of a widening gap between catchment area categories. Addition of terms for other interactions between the main effect variables did not improve the model fit significantly.

Stratified analyses confirmed the falling five year mortality in all catchment area categories (table 4). The downward trend was strongest in areas served by regional/university and county hospitals, and weakest in catchment areas of large and small local hospitals.

In a second analysis, stratified by time period, the difference in prognosis between catchment area categories was confirmed (table 5). Also, the differences increased during the two last time periods.

\section{Discussion}

Our principal finding in this study is that the prognosis in patients with rectal cancer has improved steadily over the last few decades but that those who lived in catchment areas of small local hospitals were significantly worse off than patients living in areas served by regional/university hospitals. The difference in survival is not explained by differences in either mean age or the distribution of cases over time. The improvement in survival was greatest in areas where university and county hospitals were responsible for medical services. We found no indication that the observed differences decreased, as we have previously reported for colon cancer in Sweden. ${ }^{13}$

The Swedish public health system provides equal access to health care in all hospital catchment areas. There was no organised screening for colorectal cancer in Sweden during the study period. Thus the observed differences in survival between these geographically defined populations are not likely to be explainable by differences in the stage or severity of the rectal cancer at presentation, particularly as each catchment area category represents a mix of areas from all parts of Sweden, including all levels of urbanisation. Also, it is unlikely that patients' delay in seeking health care would differ between area categories. We therefore suggest that the differences in outcome may reflect dissimilarities in the overall management of these patients. These may involve variations in diagnostic practices or in the quality of anaesthesiological or surgical management. Patients with rectal cancer typically present with local symptoms which usually prompt a standardised diagnostic work up. The tumours are accessible for examination by simple rigid rectoscopy, which is often performed by general practitioners. Thus it is unlikely that differences in diagnostic efficiency would account for more than a minor part of the differences in survival between catchment area categories. Therefore the reason for the differences seems to involve the organisation of rectal cancer care and the quality of the treatments offered.

Table 4 Relative hazard and 95\% confidence intervals (within parentheses) of dying from rectal cancer from the first five years of follow up by time period of diagnosis, stratified by hospital catchment area category

\begin{tabular}{lllll}
\hline \multirow{2}{*}{ Period } & \multicolumn{2}{l}{ Hospital catchment area category } & & \\
\cline { 2 - 5 } & Regional & County & Large local & Small local \\
\hline $1973-76$ & 1.00 (reference) & 1.00 (reference) & 1.00 (reference) & 1.00 (reference) \\
$1977-80$ & $0.86(0.77$ to 1.00$)$ & $0.83(0.72$ to 0.96$)$ & $0.91(0.79$ to 1.06$)$ & $0.91(0.79$ to 1.04$)$ \\
$1981-84$ & $0.83(0.73$ to 0.95$)$ & $0.71(0.61$ to 0.82$)$ & $0.86(0.74$ to 1.00$)$ & $0.79(0.69$ to 0.91$)$ \\
$1985-88$ & $0.67(0.58$ to 0.76$)$ & $0.70(0.60$ to 0.81$)$ & $0.82(0.70$ to 0.95$)$ & $0.76(0.66$ to 0.87$)$ \\
$1989-92$ & $0.63(0.54$ to 0.74$)$ & $0.59(0.50$ to 0.70$)$ & $0.77(0.65$ to 0.91$)$ & $0.72(0.62$ to 0.84$)$ \\
\hline
\end{tabular}

Table 5 Relative hazard and 95\% confidence intervals (within parentheses) of dying from rectal cancer from the first five years of follow up by hospital catchment area category stratified by time period of diagnosis

\begin{tabular}{|c|c|c|c|c|c|}
\hline \multirow[b]{2}{*}{ Hospital catchment area category } & \multicolumn{5}{|l|}{ Period } \\
\hline & $1973-76$ & $1977-80$ & $1981-84$ & $1985-88$ & 1989-92 \\
\hline Regional & 1.00 (reference) & 1.00 (reference) & 1.00 (reference) & 1.00 (reference) & 1.00 (reference) \\
\hline County & $1.08(0.97$ to 1.19$)$ & $1.02(0.88$ to 1.18$)$ & $0.92(0.79$ to 1.06$)$ & $1.12(0.95$ to 1.38$)$ & $1.01(0.87$ to 1.18$)$ \\
\hline Large local & $0.97(0.86$ to 1.09$)$ & $1.01(0.86$ to 1.19$)$ & $1.00(0.85$ to 1.18$)$ & $1.19(0.98$ to 1.43$)$ & $1.19(1.01$ to 1.40$)$ \\
\hline Small local & $1.10(0.98$ to 1.23$)$ & $1.13(0.98$ to 1.32$)$ & $1.04(0.90$ to 1.22$)$ & $1.24(1.04$ to 1.48$)$ & $1.27(1.09$ to 1.48$)$ \\
\hline
\end{tabular}


Although referral to specialised hospitals may be part of the care that is provided, our data indicate that only a small proportion of the patients were, in fact, referred. Besides, as patients thus referred appeared to be the sickest, as judged from their poor survival, these referrals may have had the result that the observed difference in survival between catchment area categories represented a slight underestimation of the true difference in outcome between hospital categories. Referrals of more serious cases from smaller to larger hospitals may perhaps explain why the intercategory differences in survival based upon reporting hospitals - that is, the hospitals where the patients, in most instances, were treated-were smaller than the corresponding intercategory differences based on domicile.

Previous studies of the relation between patient volumes and surgical results have been typically influenced by the case mix encountered in the hospitals under study. ${ }^{12}$ As admissions to hospitals, particularly in health care systems without strictly defined catchment areas, are preceded by a chain of decisions, on the part of the patient, the family doctor, and the hospital surgeon, patients may be selectively distributed among different categories of hospitals. Hence comparisons in hospital based studies may be confounded by the stage or severity of the disease or other prognostic factors. This may explain why previous studies led to conflicting conclusions. Even if adjustment for disease stage is attempted, the shift in stage that occurs if specialised-and presumably more experienced-surgeons and pathologists find more lymph nodes and thus more local metastases may again confound the results. Our approach compared populations rather than hospitals. Also, our design with a two decade study period and a large number of patients has not to our knowledge been applied before to studies of rectal cancer.

The steady improvement in survival that appeared to have taken place over the observation period and in all catchment area categories has also been noted in other studies. ${ }^{14-18}$ There was a noticeable difference in survival between area categories as early as one month after the rectal cancer diagnosis, and the order of rank between the categories was already clearly established by then. This short term outcome may be partly determined by the general health and nutritional status among the patients, but is less likely to be influenced by the stage or severity of the disease at diagnosis. This variation in short term results between catchment area categories is more likely to be due to differences in perioperative care. The quality of anaesthesiological management may have a greater impact on perioperative survival than the efficacy of surgical or non-surgical tumour removal.

The differences in survival between catchment area categories persisted, however, one and five years after diagnosis. Mesorectal excision, a development of the traditional surgical technique associated with better local tumour control, at least in expert hands, ${ }^{19}$ was introduced by some interested surgeons in the 1980s but did not gain widespread popularity in Sweden during the study period. Interestingly, during this period an increasing proportion of abdomino-perineal resections were replaced by anterior resections, which would seem to offer less favourable conditions for radicality.

Perioperative radiation was subject to large randomised trials at county and regional hospitals during the early and mid 1980s, ${ }^{20}{ }^{21}$ whereas only a small proportion of the patients treated at non-academic hospitals received such adjuvant treatment. ${ }^{22}{ }^{23}$ Although most previous studies of perioperative radiotherapy have failed to show an unequivocal effect on survival, ${ }^{24}$ a recent Swedish multicentre study showed a significant survival advantage after 25 Gy of preoperative radiation among patients with resectable rectal cancer. ${ }^{3}$ Hence variations in the use of perioperative radiotherapy may have contributed to the intercategory differences in survival observed in our study. More active follow up after treatment seems to have little influence on the prognosis. ${ }^{25}{ }^{26}$ This again did not differ between hospital categories.

Although Sweden had no specialised centres for rectal cancer surgery during the period under study, a concentration of such surgery to fewer hands may still have taken place within each hospital. This is seemingly contradicted by a marked increase in the number of general surgeons in Sweden during the past 20 years, but it also became increasingly common to have more than one surgeon at the operating table. We speculate that an increasing subspecialisation into oncological surgery that occurred at university hospitals during the $1980 \mathrm{~s}$ may also have contributed to the acceleration of the positive trend noted for this hospital catchment area category in the last years of our study.

The strengths of our study include the population based design with inclusion of all rectal cancers that occurred in Sweden during the study period, the high proportion of cases in which the tumours were histologically confirmed, the strictly defined catchment areas, the complete follow up regardless of catchment area category, the availability of population based expected rates of survival, and the precision of our survival estimates owing to the large numbers of cases. Our most important caveat is the assumption that the average stage of the disease at which patients with developing rectal cancer seek medical care is unrelated to the place of residence. However, with the prerequisites prevailing in Sweden, it seems far fetched to postulate the opposite.

A further strength of our study is that our data reflect the integrated effect of a whole chain of measures taken to diagnose and treat rectal cancer. We can only speculate as to which specific components of the medical services offered to the populations account for the observed differences in survival between catchment area categories. Thus, our results do not provide the necessary guidance to remedy these disturbing differences. Our findings should perhaps be seen as an alarm signal to surgeons and health care planners. Clearly, 
more in depth studies, preferably focusing on steps in the management chain, and with adjustment for disease stage at diagnosis, are warranted.

1 Sowden AJ, Grilli R, Rice N. The relationship between hospital volume and quality of health outcomes. CRD Report 8 (Part 1). NHS Centre for Reviews and Dissemination, University of York, 1997.

2 Järhult J. The importance of volume for outcome in cance surgery: an overview. Eur f Surg Oncol 1996;22:205-15.

3 Swedish Rectal Cancer Trial. Improved survival with preoperative radiotherapy in resectable rectal cancer. $N$ Engl $\mathcal{F}$ Med 1997;336:980-7.

4 Glimelius B, Isacsson, Jung B, et al. Radiotherapy in addition to radical surgery in rectal cancer. Acta Oncol 1995;34:565-70.

5 Medical Research Council Rectal Cancer Working Party. Randomised trial of surgery alone versus radiotherapy followed by surgery for potentially operable locally advanced lowed by surgery for potentially operable
rectal cancer. Lancet 1996;348:1605-10.

6 Cancer Incidence in Sweden 1993. Yearbooks. Stockholm: The National Board of Health and Welfare, 1996.

7 Cutler S, Ederer F. Maximum-utilization of the life table Cutler S, Ederer F. Maximum-utilization of the life table 710 .

8 Ederer F, Axtell I, Cutler S. The relative survival rate: A statistical methodology. Natl Cancer Inst Monogr 1961;6:10121.

9 Hakulinen T, Abeywickrama K. A computer program package for relative survival analysis. Computer Programs in Biomedicine 1985;19:197-207.

10 Hakulinen T, Tenkanen L. Testing equality of relative survival patterns based on aggregated data. Biostatistic 1987;43:313-25.

11 Hakulinen T, Tenkanen L. Regression analysis of relative survival rates. Applied Statistics 1987;36:309-17.

12 Payne CD. GLIM manual, release 3.77. Oxford: NAG, 1986

13 Blomqvist P, Ekbom A, Nyrén O, et al. Survival after colon cancer 1973-1990 in Sweden: convergence between catchment areas. Ann Surg 1997;225:208-16.
14 Chu KC, Tarone RE, Chow WH, et al. Temporal patterns in colorectal cancer incidence, survival, and mortality from 1950 through 1990. I Natl Cancer Inst 1994;86:997-1006.

15 Cancer Survival in Sweden in 1961-1991. The National Board of Health and Welfare. Centre for Epidemiology. Acta Oncol 1995;34(suppl 4):22-6.

16 McArdle CS, Hole D, Hansell D, et al. Prospective study of colorectal cancer in the west of Scotland: 10-year follow-up. Br ₹ Surg 1990;77:280-2.

17 Gordon NL, Dawson AA, Bennett B, et al. Outcome in colorectal adenocarcinoma: two seven-year studies of a population. BMF 1993;307:707-10.

18 Fändrich F, Schroeder DW, Saliveros E. Long-term survival fter curative resection for carcinoma of the rectum. $\mathcal{F} \mathrm{Am}$ Coll Surg 1994;178:271-6.

19 MacFarlane JK, Ryall RDH, Heald RJ. Mesorectal excision for rectal cancer. Lancet 1993;341:457-60.

20 Swedish Rectal Cancer Trial. Initial report from a Swedish multicentre study examining the role of preoperative irradiation in the treament of patients with resectable rectal carcinoma. Br F Surg 1993;80:1333-6.

21 Holm T, Cedermark B, Rutqvist LE. Local recurrence of rectal adenocarcinoma after "curative" surgery with and without preoperative radiotherapy. Br F Surg 1994;81:4525.

22 Järhult J, Mikkelsen I, Thulin A. [Surgery of colorectal cancer outside the regional hospitals. Good results, but internal specialization is desirable]. Lakartidningen 1995; 92:2325-8

23 Holm T, Johansson H, Cedermark B, et al. Influence of hospital- and surgeon-related factors on outcome after hospital- and surgeon-related factors on outcome after radiotherapy. Br F Surg 1997;84:657-63.

24 Buyse M, Zeleniuch-Jacquotte A, Chalmers TC. Adjuvant therapy of colorectal cancer. Why we still don't know. FAMA 1988;259:3571-8

25 Böhm B, Schwenk W, Hucke HP, et al. Does methodic longterm follow-up affect survival after curative resection of colorectal carcinoma? Dis Colon Rectum 1993;36:280-6.

26 Safi F, Link KH, Beger HG. Is follow-up of colorectal cancer patients worthwhile? Dis Colon Rectum 1993;36:63643. 